\title{
LOCALIZATIONS OF ESSENTIAL EXTENSIONS
}

\author{
by K. R. GOODEARL and D. A. JORDAN
}

(Received 8th September 1986)

In an earlier paper [4] we considered the question of whether an injective module $E$ over a noncommutative ring $R$ remains injective after localization with respect to a denominator set $X$ in $R$. A related question is whether, given an essential extension $N$ of an $R$-module $M$, the localization $N\left[X^{-1}\right]$ must be an essential extension of $M\left[X^{-1}\right]$. In [1] it is shown that if $R$ is left noetherian and $X$ is central in $R$, then localization at $X$ preserves both injectivity and essential extensions of left $R$-modules and, hence, preserves injective hulls and minimal injective resolutions. We shall observe that preservation of injectivity under localization at $X$ is implied by preservation of essential extensions under localization at $X$. In [4] positive results on the preservation of injectivity were obtained for the case where $R$ is right and left noetherian right fully bounded, and for the case where $X$ consists of regular normalizing elements. We shall show that these results can be strengthened to positive results on the preservation of essential extensions. There is an example in [4] of a simple noetherian domain $R$ with a denominator set $X$ such that localization at $X$ does not preserve injectivity. Much easier examples are available to demonstrate that localization at $X$ need not preserve essential extensions of $R$-modules even when $R$ is noetherian. We shall present two such examples, in one of which $R$ is hereditary, so that injectivity must be preserved by localization at $X$. Thus preservation of injectivity and preservation of essential extensions are not equivalent. In the second example, $X$ is the set of elements regular modulo all the prime ideals in a clique of $R$.

This research was partly done during a visit of the first author to Great Britain in May 1985, supported by a grant from the Science and Engineering Research Council.

If $X$ is a right or left denominator set in a ring $R$ and $M$ is an $R$-module, we shall write $t_{X}(M)$ and $M\left[X^{-1}\right]$ for the $X$-torsion submodule of $M$ and the $X$-localization of $M$ respectively. We begin by establishing two alternative characterizations of denominator sets which preserve essential extensions. One of these emphasizes the significance of torsion modules in this context, while the other makes it clear that preservation of essential extensions implies preservation of injectivity.

Theorem 1. Let $X$ be a right denominator set in a ring $R$. Then the following conditions are equivalent:

(i) For every essential extension $N$ of a right $R$-module $M$, the localization $N\left[X^{-1}\right]$ is an essential extension of $M\left[X^{-1}\right]$ (as either $R$-modules or $R\left[X^{-1}\right]$-modules).

(ii) Every essential extension of an $X$-torsion right $R$-module is $X$-torsion.

(iii) Every injective right $R$-module is a direct sum of its $X$-torsion submodule and an $X$-torsion-free submodule. 
Proof. (i) $\Rightarrow$ (ii) This is clear from the fact that a right $R$-module $M$ is $X$-torsion if and only if $M\left[X^{-1}\right]=0$.

(ii) $\Rightarrow$ (iii) This follows from the fact that the $X$-torsion submodule of an injective right $R$-module $E$ is essential in a direct summand of $E$.

(iii) $\Rightarrow$ (i) We may, by replacing $N$ by its injective hull, assume that $N$ is injective. By (iii), $N=T \oplus S$ where $T=t_{X}(N)$ and $S$ is $X$-torsion-free. Since $S$ is also injective, $N\left[X^{-1}\right]=S\left[X^{-1}\right]=S$. On the other hand, as $M$ is essential in $N$ we have $M \cap S$ essential in $S$, and since $M \cap S \leqq M\left[X^{-1}\right]$ we conclude that $M\left[X^{-1}\right]$ is essential in $S$.

Note that for a right noetherian ring $R$, condition (iii) of Theorem 1 can be replaced by the condition "every indecomposable injective right $R$-module is either $X$-torsion or $X$-torsion-free".

Corollary 2. Let $X$ be a right denominator set in a ring $R$, such that $X$-localization preserves essential extensions (that is, such that condition (i) of Theorem $I$ holds). Then:

(i) For every injective right $R$-module $E$, the localization $E\left[X^{-1}\right]$ is an injective right $R\left[X^{-1}\right]$-module.

(ii) For every right $R$-module $M$, the injective hull of $M\left[X^{-1}\right]$ (as an $R\left[X^{-1}\right]$-module) is $E(M)\left[X^{-1}\right]$.

(iii) For every right $R$-module $M$, the $X$-localization of the minimal injective resolution of $M$ is the minimal injective resolution of $M\left[X^{-1}\right]$.

Proof. (i) By Theorem 1, $E=T \oplus S$ where $T=t_{X}(E)$ and $S$ is $X$-torsion-free. Then $S$ is injective, and $E\left[X^{-1}\right]=S\left[X^{-1}\right]=S$. Thus $E\left[X^{-1}\right]$ is an injective right $R$-module. By [9, Exercise 12, p. 62], $E\left[X^{-1}\right]$ is also injective as a right $R\left[X^{-1}\right]$-module.

(ii) This follows from (i) and the hypothesis that $X$-localization preserves essential extensions.

(iii) This follows from (ii) and the exactness of $X$-localization.

Corollary 3. Let $R$ be a right and left noetherian right fully bounded ring, let $X$ be a right and left denominator set in $R$, and let $N$ be an essential extension of a right $R$ module $M$. Then $N\left[X^{-1}\right]$ is an essential extension of $M\left[X^{-1}\right]$.

Proof. Theorem 1.1 of [4] states that $X$-localization preserves injectivity. The argument used to prove this result proceeds by showing that every indecomposable injective right $R$-module is either $X$-torsion or $X$-torsion-free. As $R$ is right noetherian, condition (iii) of Theorem 1 follows.

Theorem 1.3 of [4] states that if $R$ is right noetherian and $X$ is a right denominator set consisting of regular normalizing elements of $R$, then localization at $X$ preserves injectivity. (Recall that a normalizing element in a ring $R$ is an element $c \in R$ such that $c R=R c$.) The next result is that localization at such a set preserves essential extensions. Together with Corollary 2, it provides an alternative proof of [4, Theorem 1.3]. 
Theorem 4. Let $R$ be a right noetherian ring, let $X$ be a right denominator set of regular normalizing elements of $R$, and let $N$ be an essential extension of a right $R$-module $M$. Then $N\left[X^{-1}\right]$ is an essential extension of $M\left[X^{-1}\right]$.

Proof. We shall show that condition (ii) of Theorem 1 is true for $X$. Let $M$ be an $X$ torsion right $R$-module, let $N$ be an essential extension of $M$, and suppose that $N$ is not $X$-torsion. For $n \in N$ let $a n n(n)$ denote the annihilator of $n$ in $R$. For each $c \in X$ there is an automorphism $\alpha_{c}$ of $R$ such that $\alpha_{c}(r) c=c r$ for all $r \in R$. Let $A=\left\{\alpha_{c} \mid c \in X\right\}$, which is a semigroup of automorphisms of $R$. Let $I=\alpha(\operatorname{ann}(n))$ be a maximal element of the set

$$
\left\{\alpha(a n n(n)) \mid \alpha \in A \text { and } n \in N \backslash t_{X}(N)\right\}
$$

of right ideals of $R$. Since $N$ is an essential extension of $M$ and $M$ is $X$-torsion, there exist $r \in R$ and $c \in X$ such that $n r \neq 0$ and $n r c=0$. Then $n c \in N \backslash t_{X}(N)$ and

$$
a n n(n) \subseteq \alpha_{c}(a n n(n c)) .
$$

Thus $\alpha(a n n(n)) \subseteq \alpha \alpha_{c}(a n n(n c))$ and so, by the maximality of $I$, we obtain

$$
\alpha(a n n(n))=\alpha \alpha_{c}(a n n(n c)) .
$$

Hence, $a n n(n)=\alpha_{c}(a n n(n c))$. But $n c \alpha_{c}^{-1}(r)=n r c=0$, whence $\alpha_{c}^{-1}(r) \in a n n(n c)$, and so

$$
r \in \alpha_{c}(a n n(n c))=a n n(n) .
$$

This contradicts the choice of $r$. Therefore $N$ is $X$-torsion, proving condition (ii) of Theorem 1, as desired.

Example 5. Let $K$ be a field of characteristic zero and let $R=A_{1}(K)=K[x][\theta ; d / d x]$ be the first Weyl algebra over $K$. Let $X=\left\{\theta^{i} \mid i \geqq 0\right\}$. Then $R$ is a right and left hereditary noetherian domain and $X$ is a right and left denominator set in $R$ such that $X$ localization preserves injectivity of modules but does not preserve essential extensions.

Proof. That $R$ is right and left noetherian is well-known (e.g. [3, Example 3, p. 440]). It is right and left hereditary by [6, Proposition 2]. Since $d / d x$ is locally nilpotent on $K[x]$, it follows from Leibnitz' Rule that for any $p \in K[x]$ there is some $j \in \mathbb{N}$ such that $\theta^{j} p \in R \theta$. Hence, for any $r \in R$ there exist $j \in \mathbb{N}$ and $s \in R$ such that $\theta^{j} r=s \theta$. It follows that $X$ is a left denominator set in $R$, and a similar argument shows that $X$ is a right denominator set. (Alternatively, that $X$ is a right and left denominator set is a special case of [4, Proposition 2.9].)

The ring $K[x]$ has a natural right $R$-module structure, with $\theta$ acting as $-d / d x$, such that $K[x]_{R} \cong R / \theta R$. The fact that $d / d x$ is locally nilpotent on $K[x]$ has the consequence that $K[x]_{R}$ is $X$-torsion. The right $R$-module structure on $K[x]$ can be extended to the rational function field $K(x)$ which is then an essential $R$-module extension of $K[x]$. But it is clear that $K(x)_{R}$ is not $X$-torsion; for example, the element $1 / x$ is not $X$-torsion. 
The fact that $X$-localization preserves injectivity of modules follows from the fact that $R$ is hereditary. For, if $E$ is an injective $R$-module, then $E\left[X^{-1}\right]=E / t_{X}(E)$ is an injective $R$-module by [7, Theorem 4.23] and an injective $R\left[X^{-1}\right]$-module by [9, Exercise 12, p. 62].

One situation where one might hope to apply a result on preservation of injectivity and essential extensions is where the localization is at a prime ideal, or, more generally and realistically, at a clique of prime ideals in the sense of [5]. We have been unable to decide whether such a localization in a noetherian ring must preserve injectivity, but for the best-known example of a nontrivial clique, we can show that essential extensions are not preserved by the localization.

Example 6. Let $R=\mathbb{C}[x, y \mid x y-y x=x]$ be the universal enveloping algebra of the two-dimensional solvable Lie algebra over $\mathbb{C}$. For $\alpha \in \mathbb{C}$ let $I_{\alpha}$ be the maximal ideal $x R+(y-\alpha) R$ of $R$. Then the clique of $I_{0}$ is the set $S=\left\{I_{n} \mid n \in \mathbb{Z}\right\}$, and the set

$$
\mathscr{C}(S)=\bigcap_{n \in \mathbf{Z}}\left(R \backslash I_{n}\right)
$$

is a right and left denominator set in $R$ such that localization at $\mathscr{C}(S)$ does not preserve essential extensions.

Proof. That $S$ is the clique of $I_{0}$ is established in $[5$, p. 148] (or see [8, Theorem 3.7]). We note that each $R / I_{\alpha} \cong \mathbb{C}$, so that, in the notation of $[5], \mathscr{C}\left(I_{\alpha}\right)=R \backslash I_{\alpha}$ and $\mathscr{C}(S)$ has the stated form. As commented in [5, p. 148], it is not hard to verify that $\mathscr{C}(S)$ is a right and left Ore set. (See also [8, Corollary 4.7]. For a short proof of a more general result, see [2, Proposition 3.3].) Since $R$ is a domain, $\mathscr{C}(S)$ is a right and left denominator set.

The polynomial ring $\mathbb{C}[y]$ has a natural right $R$-module structure, with $x$ acting as the $\mathbb{C}$-algebra automorphism $\alpha$ of $\mathbb{C}[y]$ determined by $\alpha(y)=y-1$, and with $\mathbb{C}[y]_{R}$ isomorphic to $R /(x-1) R$. Since $x-1 \in \mathscr{C}(S)$, this cyclic module is $\mathscr{C}(S)$-torsion. The $R$ module structure of $\mathbb{C}[y]$ can be extended to the quotient field $\mathbb{C}(y)$, which is an essential $R$-module extension of $\mathbb{C}[y]$. It is easily checked that

$$
\{r \in R \mid(1 / y) r \in \mathbb{C}[y]\}=y R
$$

and hence that the element $1 / y$ of $\mathbb{C}(y)$ is not $\mathscr{C}(S)$-torsion. Thus condition (ii) of Theorem 1 fails for $\mathscr{C}(S)$.

In Example 6, it would be interesting to know whether the injective hull of $R /(x-1) R$ remains injective after localization with respect to $\mathscr{C}(S)$. There is a larger but more easily describable injective module available which contains $R /(x-1) R$, and this injective module might prove easier to test. Firsi, view $R$ as the Ore extension $\mathbb{C}[y][x ; \alpha]$ (or $\mathbb{C}[y]\left[x ; \alpha^{-1}\right]$ if left-hand coefficients are preferred), set $T=\mathbb{C}(y)[x ; \alpha]$, and let $Q$ be the Goldie quotient ring of $T$. Observe that $R /(x-1) R$ embeds in $T /(x-1) T$, which in turn embeds in $Q / T$. Since $T$ is right hereditary and ${ }_{R} T$ is flat, $(Q / T)_{R}$ is injective. Therefore 
$(Q / T)_{R}$ contains a copy of the injective hull of $R /(x-1) R$. The localization $(Q / T)_{R}\left[\mathscr{C}(S)^{-1}\right]$ is also easily describable, for it is isomorphic to $(Q / A)_{R}$ where $A$ is the union of the sets $T c^{-1}$ for $c \in \mathscr{C}(S)$.

\section{REFERENCES}

1. H. Bass, Injective dimension in noetherian rings, Trans. Amer. Math. Soc. 102 (1962), 18-29.

2. A. W. Chatrers, The largest Ore set at a prime ideal: a special case, Bull. London Math. Soc. 18 (1986), 153-158.

3. P. M. СонN, Algebra, Vol. 2 (Wiley, London, 1977).

4. K. R. Goodearl and D. A. Jordan, Localizations of injective modules, Proc. Edinburgh Math. Soc. 28 (1985), 289-299.

5. A. V. Jategaonkar, Localization in Noetherian Rings (London Math. Soc. Lecture Note Series 98, Cambridge Univ. Press, Cambridge, 1986).

6. G. S. Rinehart, Note on the global dimension of a certain ring, Proc. Amer. Math. Soc. 13 (1962), 341-346.

7. J. J. Rotman, An Introduction to Homological Algebra (Academic Press, New York, 1979).

8. G. Sigurdsson, Links between prime ideals in differential operator rings, J. Algebra 102 (1986), 260-283.

9. B. Stenström, Rings of Quotients (Springer-Verlag, Berlin, 1975).

Department of Mathematics

UNIVERSITY OF UTAH

Salt Lake City, Utah 84112,

U.S.A.
Department of Pure Mathematics

UNIVERSITY OF SHEFFIELD

SHEFFIELD S3 7RH

ENGLAND 